\title{
A missed opportunity of a preventable maternal death-a case report from Sri Lanka
}

\begin{abstract}
High maternal mortality rate is one of the major public health issues in under-privileged countries. Around $94 \%$ of global maternal deaths are reported annually from these countries. Sri Lanka has achieved the lowest maternal mortality rate in the South Asian region in the year 2015. The current National Maternal Mortality Surveillance System in Sri Lanka has been instrumental in achieving this. A forty year old mother of two grownup children became pregnant after 7 years of her second marriage. She and her husband warmly welcomed the pregnancy. Her two children from the previous marriage were normal vaginal deliveries without any complication and were now aged 23 and 20 years. By 08 weeks of period of amenorrhoea (POA), she attended the local obstetric clinic where the routine examination revealed a cardiac murmur. She was referred to a tertiary care hospital for further management. By 16 weeks of POA she was diagnosed to have critical aortic stenosis, moderate aortic regurgitation with mild mitral stenosis and regurgitation with left ventricular dysfunction. The consultant obstetrician, physician, cardiologist and the anaesthetist had carefully assessed her and decided that continuation of the pregnancy poses a significant risk on her life. They also recommended her pregnancy to be therapeutically terminated which she had adamantly refused. Thereafter she defaulted the local clinic as well as the one at the teaching hospital. Around the $20^{\text {th }}$ week of POA her general health condition started to deteriorate. By the end of the $27^{\text {th }}$ week of POA she was admitted to the same teaching hospital with severe shortness of breath and extreme fatigability. An intrauterine death, pneumonia and sepsis were diagnosed clinically. Despite vigorous treatment she died the next day. The postmortem examination confirmed the clinical diagnosis and rheumatic valvular heart disease was evident at the autopsy. The authors wish to discuss the concepts of preventable maternal death, indirect maternal death and the significance of effective communication with the patients when persuading them to comply with life-saving intimate clinical decisions.
\end{abstract}

Keywords: maternal death, rheumatic valvular heart disease, preventable death, therapeutic termination of pregnancy, communication skills
Volume 7 Issue 6 - 2019

\section{Ariyarathna HTDW, Hulathduwa SR \\ Department of Forensic Medicine, Faculty of Medical Sciences, University of Sri Jayewardenepura, Sri Lanka}

\author{
Correspondence: Ariyarathna HTDW, Senior Lecturer, \\ Department of Forensic Medicine, Faculty of Medical Sciences, \\ University of Sri Jayewardenepura, Gangodawila, Nugegoda, \\ Sri Lanka,Tel +94 I I-2802030, Email ariyaratn@sjp.ac.lk, \\ weerawickramer@hotmail.com
}

Received: November 30, 2019 | Published: December 19, 2019

\section{Introduction}

Due to well-planned approaches the maternal death rate has been falling globally though certain fluctuations are noted among countries and even in the same country from year to year. ${ }^{1}$ It has been recognized that the risk of maternal death is 33 times more in less-resourced (under-developed) countries compared to resource-rich (developed) countries. ${ }^{2}$ Approximately 295000 mothers died during and following pregnancy and childbirth in the year 2017 . The vast majority of these deaths $(94 \%)$ occurred in low-resourced settings. The WHO further states that the majority of these deaths could have been prevented. ${ }^{3}$

An indirect maternal death is defined as "maternal death resulting from previously existing disease or disease that developed during pregnancy and which was not due to direct obstetric causes but which was aggravated by physiologic effects of pregnancy." Complications during pregnancy and child-birth claim over half a million lives of mothers in each year under the category of "preventable maternal deaths." ${ }^{5}$ In this article the authors wish to highlight the significance of "indirect causes" and "preventable maternal deaths" in Sri Lanka. The WHO as well as the Institute for Health Metrics and Evaluation (IHME) at the University of Washington, emphasizes the importance of addressing the indirect causes for maternal deaths. Indirect maternal deaths amount to $27.5 \%$ of all maternal deaths globally which is slightly more than one fourth of the total. The highest proportion of indirect maternal deaths had been reported from the South Asia and Sub-Saharan Africa. ${ }^{6,7}$ The percentages of direct and indirect maternal deaths in Sri Lanka had shown certain fluctuations over the past few years. In 2016, the direct maternal death rate had increased compared to that of the previous year. It was $55 \%$ of the total maternal deaths reported in 2016. Post viral diseases (Dengue haemorrhagic fever), heart diseases and gastrointestinal bleeding had been identified as the leading direct causes of death. ${ }^{8}$ As per the indirect causes, 38 deaths (out of 215) were due to complications of dengue and influenza. In the year 2017 the numbers of maternal deaths due to direct and indirect causes were 45 and 82 respectively. Similar to the year 2016, complications of dengue fever was the commonest cause and the same had been noticed in the year 2017 accounting for 21 deaths. Others causes were respiratory diseases including influenza (20 deaths in number) and malignancies (6 deaths in number).

According to Sri Lankan statistics, approximately $70 \%$ of the total maternal deaths could be categorized as preventable. Furthermore, $22 \%$ out of those "preventable" deaths were attributed to or linked 
with lack of effective contraception. ${ }^{9}$ The set goal in the context of maternal mortality is to end the preventable maternal deaths by the year 2035. ${ }^{10}$ Optimizing the therapeutic and clinical management protocols for pregnant women would certainly help in achieving this goal. Paying attention to the concerns of the expectant mother and her immediate social circle as well as effective communication of certain crucial issues and facts to the expectant mother and her husband during the entire period of gestation and immediately after the delivery too would expedite the process of achieving this target in Sri Lanka by 2035 specially when we consider the low socio-economic and educational standards of a sizeable proportion of pregnant women in Sri Lanka.

\section{Case report}

A forty year old woman only studied up to the eighth grade, a mother of two grown-up children got married for the second time and became pregnant after seven years of the second marriage. Her two children were born as uncomplicated vaginal deliveries, 23 and 20 years ago in a peripheral hospital. She had been suffering from general ill-health since two to three years prior to her death, without any obvious reason though it was not a limiting-factor for her dailypursuits. In the meantime she got pregnant for the third time in her life, after seven years of her second marriage. A heart murmur had been detected at the local obstetric clinic on the booking visit at eight weeks of POA. She was referred to the nearest tertiary care hospital for further management, where she was diagnosed to have critical-stage aortic stenosis, aortic regurgitation, mitral stenosis and regurgitation along with left ventricular dysfunction. She was assessed by a multidisciplinary panel of experts including the treating obstetrician, cardiologist, general physician and the anaesthetist and by the POA of 16 weeks she was informed regarding the therapeutic termination as the expert panel was of view that further continuation of her pregnancy would have posed a great thereat to her life. A short date was given for her to admit for the surgery as it was urgent. The husband claimed that they had not been adequately informed about the seriousness of her cardiac condition, its life-threatening nature as well as the nature of the procedure to be carried out on her and the options available other than the termination of pregnancy. He also claimed that both of them had been looking forward to having a baby and it had been a precious opportunity as she had conceived only after seven years of their marriage. Though the husband had asked her to comply with what the doctors have suggested, she had adamantly refused and had never gone to any of the two maternal clinics thereafter. It was evident from the husband's version that the breaking of the bad news and explaining the critical medical condition and its therapeutic approaches to them by the ward staff was very poor and the communication skills were very unsatisfactory.

From the $20^{\text {th }}$ week of POA onwards, her fatigability and shortness of breath gradually worsened until it warranted her to be admitted to the same ward in the same tertiary care hospital during the latter part of the $27^{\text {th }}$ week of POA in a state of severe restlessness, dyspnoea and mild fever. On admission she was in heart failure possibly due to rheumatic valvular heart disease. Abdominal ultrasound scan confirmed Intra Uterine Death (IUD). An emergency hysterotomy was performed. She developed fever and died the next day following multi-organ failure. An inquest was requested and a judicial post-mortem examination was performed by the consultant judicial medical officer. The autopsy revealed classical and full range of rheumatic valvular heart disease which is a rarity today. Figures 1-4 respectively show the valvular changes, severely congested lungs with macroscopic features of ARDS and congested brain. Her death was an indirect maternal death. Haemodynamic challenges of an advancing pregnancy would have pushed her to the verge of cardiac failure. Otherwise her rheumatic valvular changes would not have caused her death at this age.

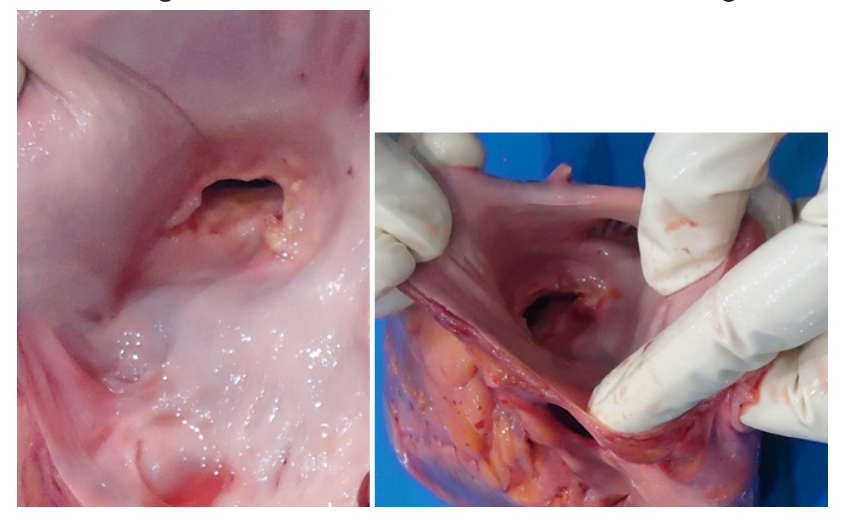

Figure I Severe mitral stenosis (fish-mouth appearance).

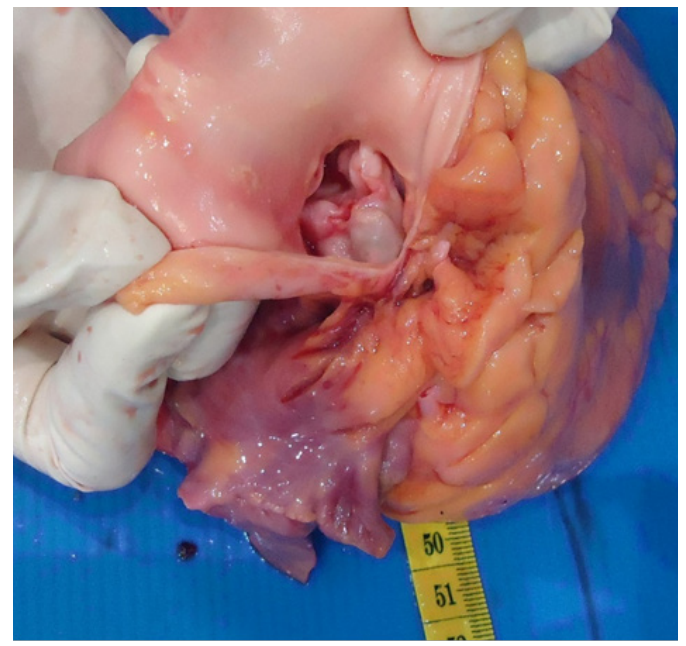

Figure 2 Note the severe aortic stenosis

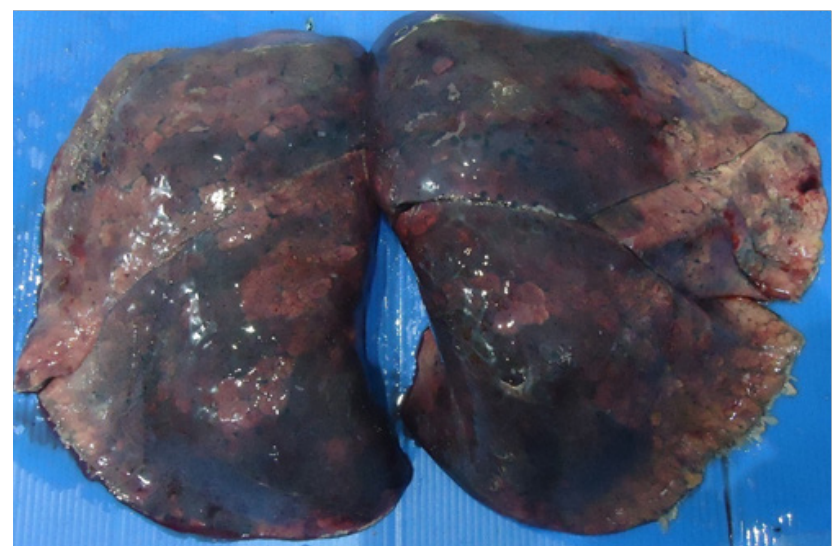

Figure 3 Severely congested lungs with macroscopic features of ARDS. 


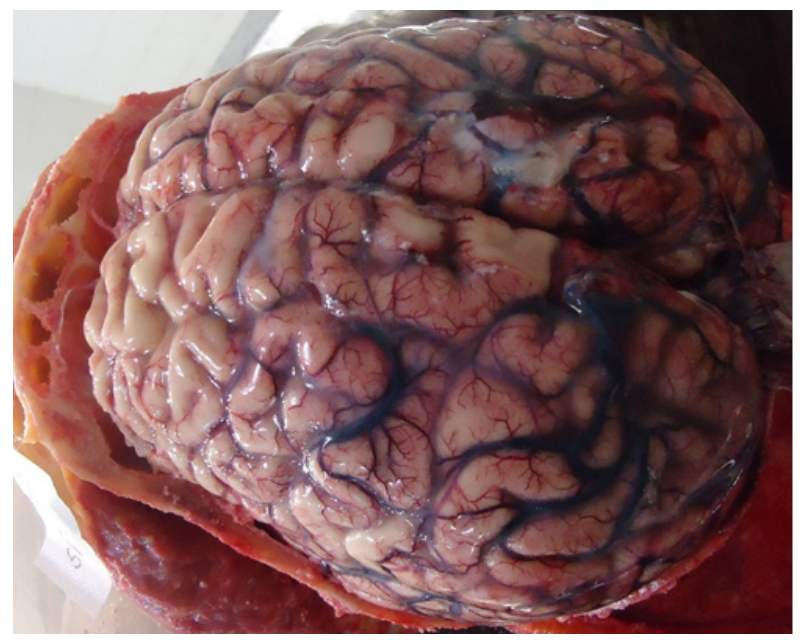

Figure 4 Severe congestion of the brain.

\section{Discussion}

It was conclusively evident that the deceased had rheumatic valvular-heart disease (RHD). She had given birth to her first and second children when she was 16 and 19 years of age respectively Both deliveries were uncomplicated normal vaginal deliveries. Until around three to four years before death she had been actively carrying out her daily routine with normal exercise tolerance. She had started to feel the progressively-worsening fatigue and shortness of breath only in the last three to four years of her life. She had apparently neglected those symptoms as they were not severe enough to limit her daily routines. Her second husband was unaware of her past medical conditions. Her two children, parents or siblings were unaware of her cardiac condition. What would have been her clinical picture at the time of the two previous deliveries? The authors can speculate three possibilities. Firstly, her cardiac condition would have been too mild to be detected. Secondly, even if her cardiac condition had been developed to a detectable level, it would possibly have been missed at the rural hospital during the first and second deliveries. Thirdly, her rheumatic valvular heart disease could have been developed in the latter years after her second delivery, which anyway is a rare possibility considering the natural history of the disease. With all such speculations, the natural history of rheumatic fever becomes a relevant issue in this case.

Since early $1900 \mathrm{~s}$ the incidence and prevalence of Acute Rheumatic Fever (ARF) and Rheumatic Heart Disease (RHD) have been decreasing in the developed world though it still is a considerable burden in the less privileged countries. ${ }^{11}$ Lesser than $0.3 \%$ of people following two to four weeks of acute pharyngitis by Streptococcus pyogenes (group A Streptococcus) can get rheumatic fever which is an overwhelming autoimmune response. ${ }^{2}$ Acute rheumatic fever causes damage to many organs including the heart, joints, brain, and skin. A severe episode of acute rheumatic fever can cause valvular damage in the heart. At times, with multiple recurrent episodes of acute rheumatic fever with carditis, it is possible to produce the entity designated as rheumatic heart disease (RHD). ${ }^{13-15}$ Although individuals of all age groups can get rheumatic fever, it is commoner among school-aged children (5-15 years of age). Rheumatic fever is very rare among children younger than three years and the elderly. This indicates that Rheumatic fever is a childhood disease and if attacks are seen in the adult life they are usually recurrences of rheumatic fever contracted during childhood rather than occurring for the first time during adulthood. ${ }^{16}$ As mentioned earlier, her second pregnancy was at the age of 19 years. Considering the natural history of RHD, the first and the second possibilities seem to be more plausible for her condition though the third possibility of her getting infected for the first time with the causative organism subsequent to her second pregnancy but prior to her third pregnancy could not be ruled out in to. ${ }^{17}$ With repeated S. pyogenes pharyngitis infections, ARF can recur and cause cumulative damage to the heart valves..$^{18}$ Symptoms of RHD may not be noticed for many years. When they do develop, symptoms depend on which heart valves are affected and the type and severity of the damage. ${ }^{19}$ By the time the deceased had been diagnosed to have RHD in early weeks of her third pregnancy, she was only marginally compromised haemodynamically. The expert panel including a consultant cardiologist after repeated and careful assessment decided therapeutic termination as the best option fore-seeing the dangers of continuation of her pregnancy. The husband described what both of them had been informed of, at her first echocardiogram and afterwards at the clinic. She had adamantly mentioned that she needed the baby at whatever the cost and bluntly refused and declined the therapeutic abortion.

It is not uncommon for a woman of her socio-economic and cultural background especially in the given socio-situational setting of becoming pregnant after seven years of marriage and at the age of 40 years closer to menopause with only a thin hope of becoming pregnant again, to adamantly refuse therapeutic abortion. According to the husband, they were informed very bluntly that the pregnancy has to be terminated with no other option. This message had been delivered to them by an assistant of the doctor who performed the echocardiogram and then by another junior doctor at the clinic. Breaking bad news is an art which needs skill and practice. Doctors do have considerable unsolicited civil authority on their patients. They can use this to persuade patients to oblige to therapeutic options which carry maximum benefit to the patient. When there is a clash between the two ethical principles of autonomy and beneficence as in this case, doctors who wish the good will of the patient should handle the situation tactfully in the best interest of the patient. In this case, if the couple had been invited to a "mini-case conference" with all four consultants of the expert panel and if the consultants themselves had explained to the deceased that continuation of the pregnancy would certainly endanger the life of the mother as well as the baby, that even though she did not feel unwell and unfit at the early stage of pregnancy, advancement of pregnancy would cause lots of changes in her body so that her heart with diseased valves would not be able to cope, that since she was at the early stages of pregnancy there may be a chance to refer her to a cardio-thoracic surgeon and consider alternatives like mechanical replacement, bio-prosthetic replacement or valve repair surgery, and that even if this pregnancy could not be preserved with cardiac surgical measures she could still undergo the same surgery following termination of the present pregnancy and become pregnant again under safe hands; she would not have "run-away" from doctors and hidden herself till she became terminally and intractably ill. As practicing consultant forensic pathologists and forensic clinicians for more than two decades it is the general understanding of the authors that most doctors do try to provide their clients with the best possible care genuinely expecting the best interest of the client. Yet, there are 
occasional situations where doctors themselves do not realize that "communication barriers" and "communication failures" prevent them from doing the expected good to their patients. What is lacking is something beyond empathy which is merely the ability to understand and share the feelings of the patient. Even if the doctor is empathetic, if he cannot explain leisurely, the problems the patient is having with suitable treatment options in a language and terminology that are best understood by the patient, sometimes being slightly assertive, directive and even authoritative, still giving due respect to patientautonomy; the doctor may fail in achieving patient compliance in complicated scenarios as this case under discussion. In this type of scenarios there is always the component of "contributory negligence" by the patient. Yet, what concerns the authors is whether this so-called contributory factor from the patient's side could have been prevented with effective communication by the medical staff.

Based on recent research some speculate that the overemphasis on direct obstetric causes for maternal deaths is the current global trend. Similarly, there is a compelling need to investigate into indirect maternal deaths as they show a rising trend. ${ }^{20}$ The term obstetric transition describes the gradual decrease in maternal mortality rates across the globe and the shifted pattern from direct causes to indirect causes under the preventable maternal deaths. This also highlights the importance of strategies to further decrease the preventable deaths. ${ }^{21,22}$

To consider a given medical condition under indirect maternal deaths, if the woman had not been pregnant she would not have died at that point from that particular medical condition. RHD is one of the best examples for this category. There are avoidable or modifiable factors identified in relation to preventable maternal deaths. Such factors could be potentially avoidable if the timely management had been done. The term "missed opportunity" is also counted under potentially avoidable maternal deaths. The authors wish to categorize the case under discussion as a "missed opportunity". There would have been a chance to survive had the doctors paid more attention to prevent a 'communication mishap' at least providing the patient with some hope with alternative measures as mentioned above. ${ }^{23}$

Every maternal death is subject to a fact finding enquiry by the Ministry of Health in Sri Lanka where it is mandatory for the obstetrician and the forensic pathologist to participate. In any enquiry into a maternal death, it is very important to identify the possible and probable avoidable factors and missed opportunities. This knowledge helps to avoid similar deaths in future. This case under discussion is a potentially avoidable death, though the patient's attitude, ignorance other socio-situational factors too had played a major role. The public health midwife (PHM) is the grass-root level care-giver of all pregnant mothers who is entitled and expected to periodically visit their homes to look into their well-being. Repeated local clinic defaults at the $\mathrm{MOH}$ (medical officer of heath)'s office conducted by the MOH and the PHM of the area were not detected and the same PHM who is supposed to pay home visits too has not taken any measures to detect and act upon this situation. It would have been prudent for the $\mathrm{MOH}$ and the PHM to wonder why this particular mother after being referred to a tertiary care hospital following detecting a murmur has never come back to the local clinic. This elaborates the lapses in the delivery system of primary care to expecting mothers which is also a contributory factor in this "avoidable" death.

\section{Conclusion}

Sri Lanka is has a reputation for strenuous work performed so far in order to lessen maternal deaths. A considerable percentage of such deaths is preventable at different levels. A multiplicity of complex and intertwined socio-situational, economic and cultural factors complicates the primary and secondary health care delivery system in avoidable maternal deaths. Though the state health care system is functioning with limited resources and manpower and already over-burdened with ever-increasing numbers of patients, the right of information by the patient and the luxury of effective communication should not be compromised. Effective communication and conveying salient facts even amidst extremely busy clinical settings is a way forward to avoiding medically preventable deaths. This would also contribute towards the WHO goal of ending preventable maternal deaths in future.

\section{Acknowledgments}

None.

\section{Ethical consent}

None.

\section{Conflicts of interest}

The author declares there are no conflicts of interest.

\section{References}

1. World Health Organization. Haywood L Brown MJ, Maria J S. Maternal Mortality. 2018.

2. WHO. Maternal mortality. 2015 .

3. WHO, UNICEF, UNFPA, et al. Maternal mortality: Levels and trends: 2000 to 2017. 2019;1-104.

4. World Health Organization. The WHO application of ICD-10 to deaths during pregnancy, childbirth and the puerperium: ICD-MM. 2012.

5. Hunt P, Mesquita J. Reducing Maternal Mortality The Contribution of the right to the highest attainable standard of Health. 2010.

6. Say L, Chou D, Gemmill A, et al. Global causes of maternal death: a WHO systematic analysis. Lancet Glob Health. 2014;2(6):323-333.

7. Kassebaum NJ, Bertozzi Villa A, Coggeshall MS, et al. Global, regional, and national levels and causes of maternal mortality during 1990-2013: a systematic analysis for the Global Burden of Disease Study 2013. Lancet. 2014;384(9947):980-1004.

8. Manushi Silva. Sri Lanka confirmed as having lowest maternal deaths in South Asia. 2019

9. Wijesinghe PS, Jayaratne K, Peiris D. National Maternal Death surveillance and Response: Sri Lankan scenario. Ceylon Medical Journal. 2019;64(1):1-3.

10. Vincent Fauveau Britt Mckinnon Yves Bergevin. Seminars in Reproductive Medicine. 2015;33(1):1-53.

11. Seckeler M, Hoke T. The worldwide epidemiology of acute rheumatic fever and rheumatic heart disease. Clinical Epidemiology. 2011;3:6784.

12. Rheumatic Fever. Retrieved from Texas Heart Institute. 
13. Rheumatic heart disease (RHD) is the most significant sequela of Rheumatic Fever (RF).

14. Gerber MA, Baltimore RS, Eaton CB, et al. Prevention of Rheumatic Fever and Diagnosis and Treatment of Acute Streptococcal Pharyngitis: a scientific statement from the American Heart Association Rheumatic Fever, Endocarditis, and Kawasaki Disease Committee of the Council on Cardiovascular Disease in the Young, the Interdisciplinary Council on Functional Genomics and Translational Biology, and the Interdisciplinary Council on Quality of Care and Outcomes Research: endorsed by the American Academy of Pediatrics. Circulation. 2009;119(11):1541-1551.

15. Mark R Wallace MD, FACP, FIDSA. Rheumatic Fever: Background, Pathophysiology, Epidemiology. Emedicine.medscape.com. 2019.

16. Newman T, Judith Marcin M. Rheumatic fever: Causes, symptoms, and treatment. Medical News Today. 2019

17. Farrell A, Zaphiropoulos G. First attack of rheumatic fever in an adult: the case for greater awareness. Annals of the Rheumatic Diseases. 1990;49(12):1008-1009.
18. Martin WJ, Steer AC, Smeesters PR, et al. Post-infectious group A streptococcal autoimmune syndromes and the heart. Autoimmunity Reviews. 2015;14(8):710-725.

19. What is Rheumatic Heart Disease? Rheumatic Heart Disease Australia 2019.

20. Nair M, Nelson Piercy C, Knight M. Indirect maternal deaths: UK and global perspectives. Obstetric Medicine. 2017;10(1):10-15.

21. Souza JP, Tunçalp O, Vogel JP, et al. Obstetric transition: the pathway towards ending preventable maternal deaths. BJOG. 2014;121(Suppl $1): 1-4$.

22. Chaves Sda C, Cecatti JG, Carroli G, et al. Obstetric transition in the World Health Organization Multicountry Survey on Maternal and Newborn Health: exploring pathways for maternal mortality reduction. Rev Panam Salud Publica. 2015;37(4-5):203-210.

23. Maternal mortality. Saving Mothers and Babies: 2. Maternal mortality. 